\title{
Revelations from Historical Experiments on Constant Speed of Light
}

\author{
Xianming Meng \\ Research School of Physics, Australian National University, Canberra, ACT, Australia \\ Email: xianming.meng@anu.edu.au
}

How to cite this paper: Meng, X.M. (2022) Revelations from Historical Experiments on Constant Speed of Light. Journal of Applied Mathematics and Physics, 10, 623-640. https://doi.org/10.4236/jamp.2022.103045

Received: January 4, 2022

Accepted: March 4, 2022

Published: March 7, 2022

Copyright $\odot 2022$ by author(s) and Scientific Research Publishing Inc. This work is licensed under the Creative Commons Attribution International License (CC BY 4.0).

http://creativecommons.org/licenses/by/4.0/

\begin{abstract}
The postulate of constant speed of light for all reference frames is the foundation of relativity theories. The resultant time dilation and length contraction are generally accepted but still perplexing for most people. By re-examining the historical evidence, this paper confirms Einstein's postulate and reveals the mechanism for the constant speed of light: the interaction between photons and matter can impart the speed of an inertial frame to the photons and thus result in the same speed of light for different inertial reference frames. This mechanism can consistently explain all major experiments on the constant speed of light.
\end{abstract}

\section{Keywords}

Photon Matter Interaction, Special Relativity, Fizeau Experiment, Michelson-Morley Experiment, Hoek Experiment, Sagnac Experiment

\section{Introduction}

There are many historical experiments that relate to the postulate of the constant speed of light in a vacuum and the speculation of the existence of "aether" for light transmission. Among them, the 1851 Fizeau water tube experiment [1] (Fizeau, 1851) and the 1887 Michaelson-Morley experiment [2] (Michael and Morley, 1887) played an important role in the aether debate and in the formation of the relativity theory. Maxwell's electromagnetic wave theory required the medium aether to propagate light, and the phenomenon of stellar aberration indicated a stationary aether; however, the Fizeau water tube experiment implied a partially dragged aether. Either way, if aether exists, it cannot be dragged fully by the earth's movement and thus should result in an aether wind as the earth rotates around the sun; however, the Michelson-Morley (MM) experiment showed 
that the predicted aether wind that would be caused by the earth's rotation around the sun cannot be detected.

Many theories have been developed to explain these experiments. The totally dragged aether theories of Stokes [3] (1845) and Hertz [4] (1890) and the emission theory of Ritz [5] [6] (1908) can explain the MM experiments perfectly, but they are unable to explain the Fizeau water tube experiment. On the other hand, the partial dragging coefficient proposed by [7] Fresnel (1818) can explain the Fizeau experiment well but not the MM experiment. Based on the null results from the MM experiment, Einstein concluded that aether does not exist. He postulated that the speed of light in a vacuum is constant for all reference frames and is independent of the speed of the light source. Based on the Lorentz transformation, he developed the special relativity theory.

Half of Einstein's postulate that light speed is independent of the speed of the light source has been proved by many experiments and observations, however, the other half of the postulate that the speed is the same for all reference frames has not yet been fully confirmed, and it is a perplexing postulate unless one accepts concepts such as length contraction and time dilation. This paper examines this part of the postulate by investigating historical experiments that have been carried out to examine the constant speed of light. The investigation confirms or proves Einstein's postulate and reveals the mechanism that results in the constant speed of light for all inertial frames.

A word of warning to the reader: The paper requires considerable patience and concentration to follow the detailed, careful, logical reasoning. Without attention to the detail, observations and experiments can be explained in different ways and therefore draw different conclusions. We might still happily accept Aristotle's claim that a heavier object falls faster than a lighter one if we had not paid attention to the friction force of the air. We might also still believe the earth-central theory if we had not paid attention to the detailed observations and accurate measurement of stellar movements. Only careful reasoning on detailed facts can reveal the truth. Much patience is required when reading this paper also because some of the logical reasoning related to the experiments is very subtle.

\section{A New Interpretation of the Fizeau Water Tube Experiment}

The 1851 Fizeau water tube experiment, which has been repeated by others, including Michaelson and Morley [8] (1886), was originally designed to test the convection of aether in water. Since aether is proven by the 1887 MichelsonMorley experiment to be purely imaginary, the water tube experiment revealed how the speed of the medium affects the speed of light.

The Fizeau experiment apparatus is illustrated in Figure 1. The tube is filled with water, which flows at speed " $w$ " in the direction shown by the broad arrows. The incoming light is split into two at the beam splitter and the two beams eventually come back to the splitter and are detected by a phase detector. While 


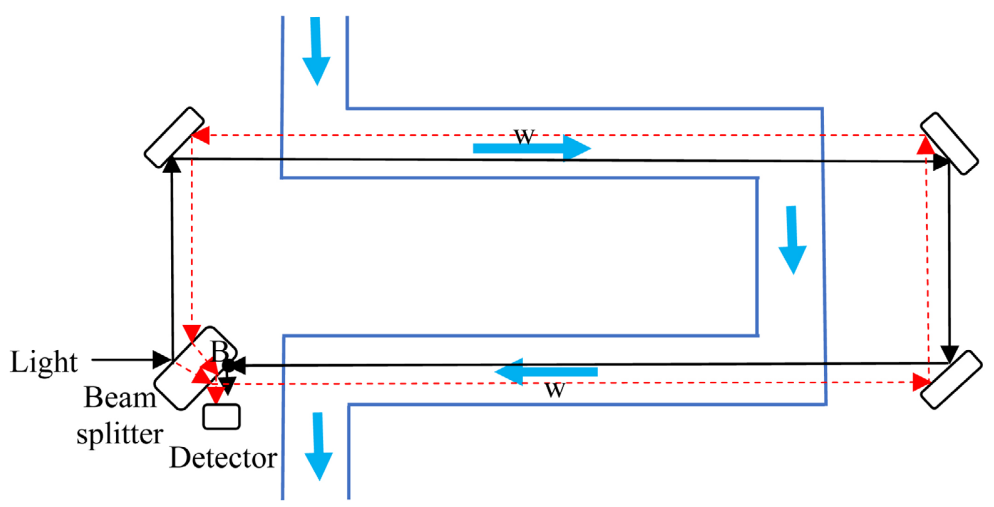

Figure 1. Setting of the 1851 Fizeau water tube experiment.

the light beam with the dashed arrows travels against the water flow and reaches the interference detector, the light beam with the solid arrows travels in the direction of water flow and reaches the interference detector. The impact of water speed can be shown by the interference pattern. If the water movement has no impact on the light speed (or the original hypothesis that the aether is stationary), the two light beams will travel the same distance at the same speed to reach the detector simultaneously, thus no interference pattern appears. On the other hand, if the water speed affects the light speed (or the original hypothesis that the aether fully or partially moves along the water flow), the two light beams travel the same distance at different speeds and thus an interference pattern will appear. The size of the impact of the water speed can be determined by examining the displacement of the interference fringes.

Let $v_{o}$ be the light speed in the stationary water, $c$ the light speed in the vacuum, and $n_{0}$ the refractive index of the stationary water. The refractive index satisfies:

$$
n_{0}=c / v_{0}
$$

If the water speed is fully absorbed by the light, e.g. the totally dragged aether theories of Stokes (1845) [3] and Hertz (1890) [4], and the emission theory of Ritz (1908) [5] [6], the speed of light in the direction of the water flowing at speed " $W$ " is:

$$
v_{1}=v_{0}+w
$$

Similarly, for the light beam moving against the water flow, the speed is:

$$
v_{2}=v_{0}-w
$$

Given the distance " $D$ " that both light beams travel in the water tube, the time each beam travels is $D / v_{I}$ and $D / V_{2}$. The equivalent distance in the vacuum is $c D / v_{I}$ and $c D / v_{2}$, respectively, so the difference in the equivalent distance that determines the interference pattern is:

$$
\Delta D=\frac{c D}{v_{2}}-\frac{c D}{v_{1}}=c D\left(\frac{1}{v_{0}-w}-\frac{1}{v_{0}+w}\right)=\frac{2 D w c}{v_{0}^{2}-w^{2}}
$$

Since $w \ll v_{0}$, then 


$$
\Delta D \approx \frac{2 D w c}{v_{0}^{2}}=\frac{2 D w}{c} \frac{c^{2}}{v_{0}^{2}}=2 D(w / c) n v_{0}^{2}
$$

This result indicates that the phase difference should be proportional to $n_{0}^{2}$. Plugging in relevant experimental setting numbers, Fizeau calculated that the displacement of the interference fringes should be $N=\Delta D / \lambda=0.4597$. However, his experimental result was $N=0.23016$, so the experiment clearly rejected all theories that indicate that light can fully absorb the water speed.

Fresnel (1818) [7] proposed a partial dragged aether theory that assumed that the light speed in the direction of the water flow is:

$$
v_{1}=v_{0}+w * f, \text { with } f=1-1 / n_{0}^{2}
$$

where $f$ is the dragging coefficient, $0<f<1$.

Based on Fresnel's theory, Fizeau calculated the expected experimental outcome:

$$
\Delta D=\frac{c D}{v_{2}}-\frac{c D}{v_{1}}=c D\left(\frac{1}{v_{0}-f w}-\frac{1}{v_{0}+f w}\right)=\frac{2 D w f c}{v_{0}^{2}-f^{2} w^{2}}
$$

After approximation, Fizeau had:

$$
\Delta D \approx \frac{2 D w}{c} \frac{c^{2}-v_{0}^{2}}{v_{0}^{2}}=2 D\left(\frac{w}{c}\right)\left(n_{0}^{2}-1\right)
$$

This calculation gave a displacement of $N=0.2022$, which is close to the experimental results; therefore, Fizeau concluded that the experiment supported Fresnel's theory.

However, history has shown again and again that theories supported by some experiments could be wrong. Fresnel's partial aether dragging theory falls into this category (although the Fresnel dragging coefficient is still valid) because the 1887 Michelson-Morley experiment proved that the aether does not exist. New theories or interpretations appeared to explain the Fizeau experiment, including Thomson (1880) [9], Lorentz (1892, 1895) [10] [11] and, finally, Laue (1907) [12] based Einstein's special theory of relativity. However, all explanations involve many assumptions or approximations.

Next, we re-examine the Fizeau experiment and provide an explanation that involves only the interpretation of both the refractive index and the Galilean principle of relative speed. The examination reveals the role of the interaction between photons and matter in the experiment.

The fact that the speed of light in a medium is less than that in a vacuum can be explained by the resistance caused by the photons bumping/scattering particles in the medium. At the microscopic level, the reduction of light speed in a medium can be explained by either the extra distance the light travels or the time taken for photon absorption and re-emission resulting from photon matter collision/scattering. The actual microscopic mechanism of photon collision or scattering with medium particles is not discussed because we are only concerned with the effect of the scattering on the speed of light. 
Let $N_{m}$ be the number of particles/molecules per unit length of the medium, a the photon-molecule scattering rate, and $e$ the decrease in light speed caused by the scattering. The resistance of a unit length of stationary water to light travel can be expressed as $e a N_{m}$. For simplicity, we assume a constant rate of photon emission from the light source, so a can be viewed as constant. We also assume that the extra distance the light travels or the absorption and re-emission time due to each scattering is unchanged, so $e$ is also constant.

As shown in panel (a) of Figure 2, light speed $v_{0}$ in a stationary medium can be expressed as the distance light travels in 1 second. For this distance of light travel, the speed reduction caused by the stationary water molecules can be expressed as $e a N_{m} * v_{0}$, so we have:

$$
v_{0}=c-e a N_{m} * v_{0}
$$

Based on the refractive index relation $n_{0}=c v_{\mathscr{\rho}}$ the above equation can be rewritten as:

$$
e a N_{m}=n_{0}-1
$$

The Fizeau experiment has shown that when the water starts to move, the light can travel at speed $v_{l}$, which is greater than $v_{0}$ but less than $v_{0}+w$, as shown in panel (b).

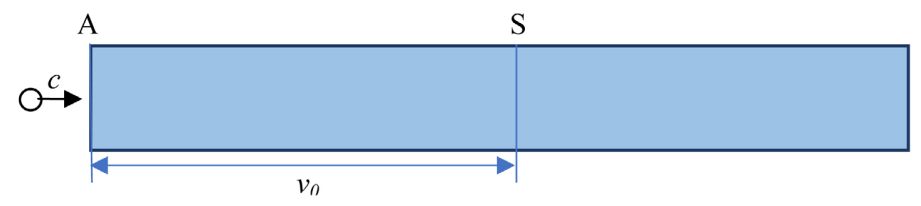

(a)

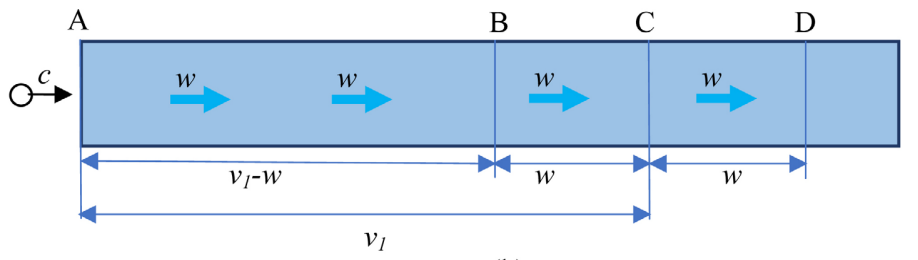

(b)

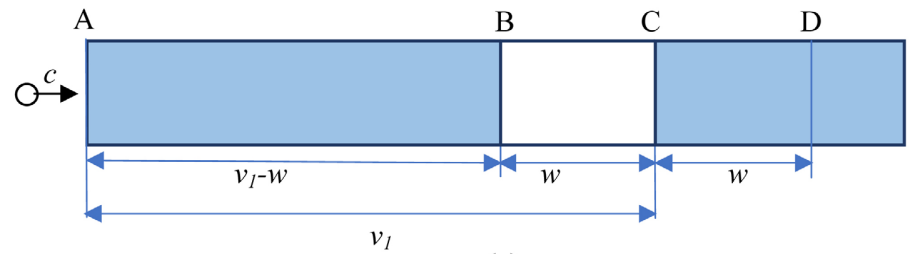

(c)

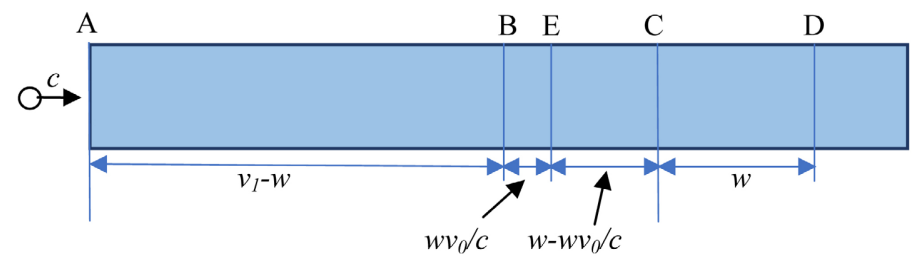

(d)

Figure 2. Decomposition of the light speed in moving water. 
Since the water molecules at the right of cross-section $\mathrm{B}$ move to the right and always stay ahead of the photon (when the photons move to $\mathrm{C}$, the molecules in section $\mathrm{BC}$ have already moved to section $\mathrm{CD}$ ), only the water from $\mathrm{A}$ to $\mathrm{B}$ (of the length $V_{l}-w$ ) is covered by the light in 1 second. Putting it differently, the relative speed of photons with respect to water flow is $V_{l}-W$, so the photons travel a distance of $V_{l}-W$ in 1 second just to catch the water molecules originally at cross-section B. Consequently, the photons travelled a distance $v_{l}$ (from A to C) in the water tube, but covered a shorter length of water $v_{l}-w$ (from A to B). As such, we have the following equation:

$$
v_{1}=c-e a N_{m} *\left(v_{1}-w\right)
$$

Plugging Equation (4) into Equation (5), we have:

$$
v_{1}=c-\left(n_{0}-1\right) *\left(v_{1}-w\right)=c-n_{0} v_{1}+v_{1}+\left(n_{0}-1\right) w
$$

or

$$
v_{1}=c / n_{0}+\left(n_{0}-1\right) w / n_{0}=v_{0}+\left(1-1 / n_{0}\right) w
$$

The above equation indicates that the water flow can increase the light speed by the amount of $\left(1-1 / n_{0}\right) w$. This effect is caused by the fewer photon-molecule collisions occurring in the shorter length of water covered by the light, compared with the case of the stationary water.

The above discussion, however, has a limitation. Equation (5) provides a picture where the light travels through a section of stationary water, $\mathrm{AB}$, which is somehow stretched to the length of AC. It has taken into account the decreased number of photon scatterings with water particles but has not reflected the impact of the velocity of the molecules in the water flow. To evaluate this impact, we must find a case where the results for stationary water are equivalent to the results for water flow. As the outcome of light travel in water flow is that the light travels from $\mathrm{A}$ to $\mathrm{C}$ but covers only the length of water from $\mathrm{A}$ to $\mathrm{B}$, the equivalent stationary case is that the light travels in the stationary water from $\mathrm{A}$ to $B$ and then travels in a vacuum from $B$ to $C$. This equivalent case is demonstrated by panel (c).

Then, we need to recall the fact that in the experiment the light never travelled in a vacuum but travelled in moving water, therefore the effect of light travelling in the vacuum BC must be brought about by the movement of the water. Water movement can contribute to the extra distance $\mathrm{BC}$ of light travel through two channels. Channel-1 is the reduced number of photon-molecule collisions due to the shortened length of water travelled by the light. According to Equation (6), the extra distance of light travel caused by channel-1 is $w\left(1-1 / n_{0}\right)$, i.e. EC in panel (d). Channel-2 is the speed of the moving water molecules imparted to the photons during the photon-molecule collision. This channel explains the remaining distance of $\mathrm{BC}$, i.e. $\mathrm{BE}$ in panel (d):

$$
\mathrm{BE}=\mathrm{BC}-\mathrm{CE}=w-w\left(1-1 / n_{0}\right)=w / n_{0}
$$

Since Channel-2 increases the light travel by the distance of BE when the light 
travelled the total distance of $\mathrm{BC}$ in 1 second, according to the definition of speed, the distance $\mathrm{BE}$ is the extra light speed gained from the moving water molecules, namely:

$$
\Delta v=\mathrm{BE}=v_{0} w / c=w / n_{0}
$$

The above equation indicates that the speed imparted to the photons through photon matter scattering is proportional to the speed of the matter and inversely related to the refractive index of the medium in which the light travels. As will be seen later, this revelation of photon matter scattering has important implications for other experiments on the constant speed of light.

With this revelation, we can improve Equation (5) by taking care of the moving water effect. The distance expressed by Equation (7) is also caused by the movement of water, so this distance should be included as part of the water effect. Thus, term $w$ in Equation (5) should be updated to $w+v_{0} w / c$. Putting it differently, the distance of light travel shown in Equation (7) is helped by the photon interaction with moving water, so this distance must be excluded when calculating the resistance caused by repetitive collision (indicated by refractive index), i.e. distance $v_{0} w / c$ should be further deducted from the term $v_{l}-w$ in Equation (5). As such, we have:

$$
v_{1}=c-e a N_{m} *\left(v_{1}-w-v_{0} w / c\right)
$$

Plugging Equations (4) and (1) into Equation (8), we have:

$$
n_{0} v_{1}=c+\left(n_{0}-1\right)\left(1+1 / n_{0}\right) w
$$

or

$$
v_{1}=v_{0}+\left(1-1 / n_{0}^{2}\right) w
$$

The above equation shows that water flow has the effect of partially dragging the light, and the dragging effect fraction is equal to the dragging coefficient proposed by Fresnel (1818) [7]. This coefficient was proved by Fizeau (1851) [1]. Michaelson and Morley's experiment (1886) [8] further confirmed this formula at higher accuracy for both water and for air flow. More importantly, they showed that the results did not depend on the length of tube, which indicated that the impact on the speed of photons did not depend on the number of photon medium scattering. In other words, as long as photon medium scattering occurs, photon can pick up the speed of the medium.

\section{Re-Examination of Michelson-Morley Experiment and Kennedy-Thorndike Experiment}

The water tube experiment of Fizeau (1851) [1] demonstrated that if aether exists, it will not be dragged fully by the medium. Based on this, Michelson and Morley reasoned that because the earth moves at $30 \mathrm{~km} / \mathrm{s}$ around the sun, the existence of aether would cause an aether wind to blow in the opposite direction to the earth's movement. Michelson and Morley (1887) [2] designed an experiment to detect aether wind. The predicted results for the Michelson-Morley experiment with and without aether wind are illustrated in Figure 3. 


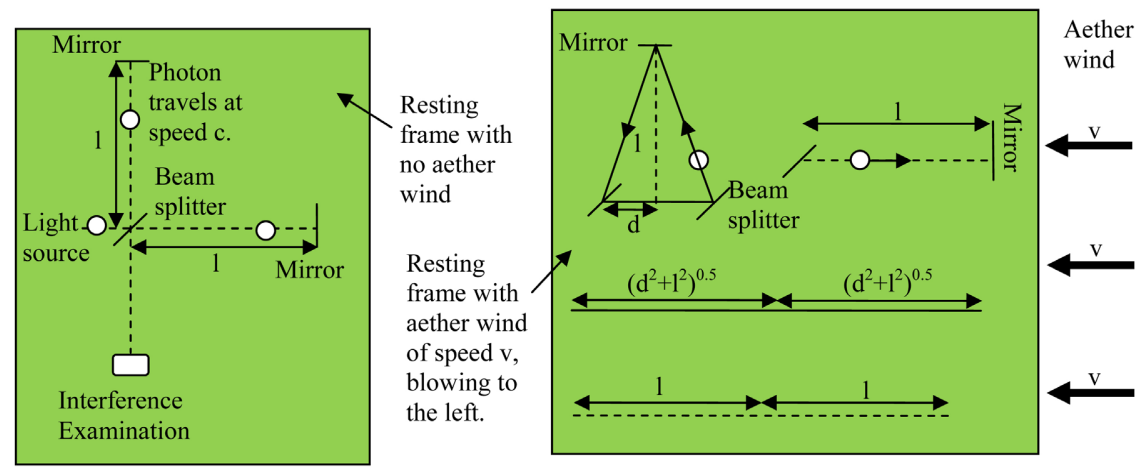

Figure 3. Aether wind interpretation of Michelson-Morley experiments.

As shown in the left panel, without an aether wind, the two photons that start from the beam splitter will travel, horizontally or vertically, the same length $I$ at the same speed $c$. After the same amount of time $2 * 1 / c$, they both come back to the beam splitter simultaneously, thus causing no interference. However, if aether exists and causes an aether wind to blow to the left at a speed of $v$, as shown in the right panel, the time for the photon to move to the top mirror and to be reflected back to the splitter will be different from the time for the photon to move to the right mirror and back to the splitter.

For the photon moving to the top mirror, while it travels up for distance $l=c t$, it will be blown to the left by a distance of $d=v t$. From this, it can be derived that $l / d=c l v$, or $v=c d l l$. In aggregate, the photon travels a distance of $2\left(d^{\mathbb{R}}+\not{P}\right)^{0.5}$ at speed $\left(c^{2}+v^{2}\right)^{0.5}$, and the total travel time is:

$$
t 1=\frac{2\left(d^{2}+l^{2}\right)^{0.5}}{\left(c^{2}+v^{2}\right)^{0.5}}=\frac{2\left(d^{2}+l^{2}\right)^{0.5}}{\left[c^{2}+\left(\frac{d c}{l}\right)^{2}\right]^{0.5}}=\frac{2 l}{c}
$$

This result is the same as that in a rest frame.

The photon moving to the right will travel a distance of $l$ at speed $c-v$ towards the right mirror and then be reflected back to the splitter at a speed of $c+v$, so its total travel time is:

$$
t 2=\frac{l}{c+v}+\frac{l}{c-v}=\frac{2 c l}{c^{2}-v^{2}}=\frac{2 l}{c-v^{2} / c}
$$

Apparently, $t 1 \neq t 2$ as long as $V \neq 0$. This will cause a phase difference and thus produce an interference pattern. If the direction of the apparatus changes, $t 1$ and $t 2$ will change and thus result in a shift in fringes. Michelson and Morley changed the direction of their apparatus and tested at different places, but they found that the shifts of the fringes were far below the theoretical calculation. The conclusion from this experiment is that aether does not exist.

However, most people tend to interpret the aether wind in the MichelsonMorley experiment as a different reference frame, so the negative experiment result is viewed as evidence of the constant speed of light for two different reference frames. This kind of interpretation is flawed because it ignores the interac- 
tion between photons and the environment.

The effect of a change of reference frame in the Michelson-Morley experiment is shown in Figure 4. As before, the left panel shows the case of the experiment conducted in a stationary frame: both photons travel for time $2^{*} 1 / c$ and come back to the beam splitter at the same time. The right panel shows the experiment done in an inertial frame moving towards the right at speed $v$.

As the frame moves horizontally, it will not affect the speed of the photons travelling to the top mirror. However, the frame movement will add to the photons a relative speed of $v$ in the horizontal direction-as in the case of star aberration. In other words, if the moving frame is used as a reference frame, i.e. treated as stationary, the speed of light towards and reflecting from the top mirror is:

$$
v^{\prime}=\left(c^{2}+v^{2}\right)^{0.5}
$$

Using this revised light speed and recalling that $v=c^{*} d / l$, the total travel time for the photons moving towards and reflected back from the top mirror can be calculated as before:

$$
t 1^{\prime}=\frac{2\left(d^{2}+l^{2}\right)^{0.5}}{\left(c^{2}+v^{2}\right)^{0.5}}=\frac{2\left(d^{2}+l^{2}\right)^{0.5}}{\left[c^{2}+\left(\frac{d c}{l}\right)^{2}\right]^{0.5}}=\frac{2 l}{c}
$$

The speed of the photons moving horizontally will be affected by the speed of the frame. While the reference frame is moving, there is no relative movement between the medium (air) and the apparatus (they all move at the same speed with the reference frame), so the amount of scattering is not affected by the speed of the reference frame and Equation (6) derived from the water tube experiment is not applicable. However, based on Equation (7), we know that the photons can pick up some of the speed of the moving matter through scattering. In the Michelson-Morley experiment, photons scatter with the moving beam splitter, mirrors and air, so the speed of the reference frame can be transferred to the photons. According to Equation (7), the speed transferred to the photons through scattering is:

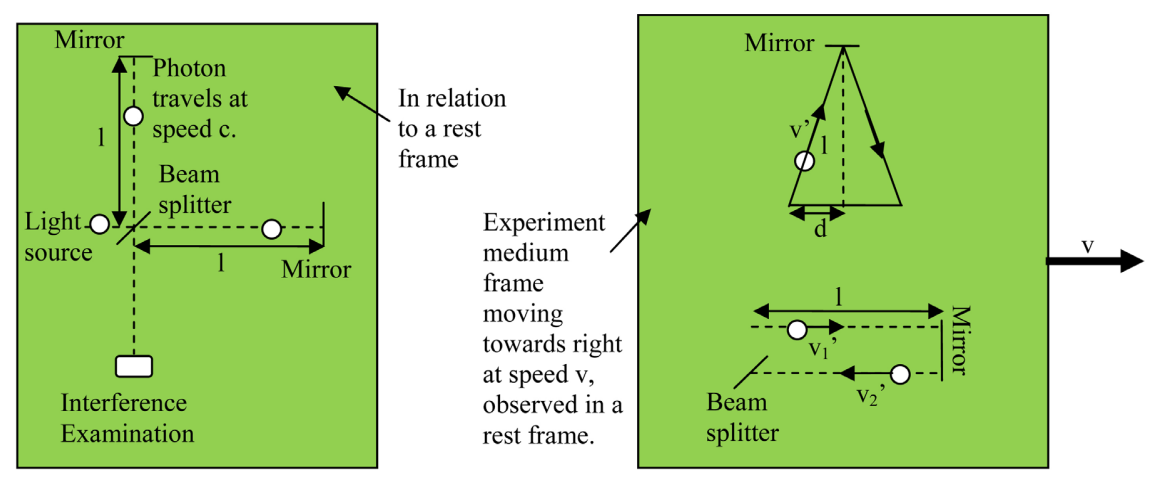

Figure 4. Reference frame interpretation of Michelson-Morley experiments. 


$$
\Delta c=v / n_{0}
$$

Since the Michelson-Morley experiment is generally conducted in air or in a vacuum, the refractive index is $n_{0}=1.00029$ for air or $n_{0}=1$ for a vacuum, so we have $c_{1}^{\prime}=c-v$ for photons moving to the right and $c_{2}^{\prime}=c+v$ for photons reflected back to the left. Substituting the value of $c$ in Equation (10) with $c_{1}^{\prime}$ and $c_{2}^{\prime}$ for the photons moving to the right and to the left, respectively, we have:

$$
t 2^{\prime}=\frac{l}{c-v+v}+\frac{l}{c+v-v}=\frac{2 l}{c}
$$

This travel time is the same as that for the photons reflected back by the top mirror (with the specified values for the refractive index, $t 1^{\prime}=t 2$ ), so there should be no fringe. In an experiment, fringes may appear for other reasons; however, if the direction of the apparatus changes, there will be no shift in fringe as $t 1^{\prime}$ and $t 2$ ' do not depend on the speed of the inertial frame. This result is consistent with the Michelson-Morley experiment.

If one ignores the interaction between the medium/mirrors and the photons, the speed of the photons will be $c$. Then the Galilean transformation can indeed lead to a shift of fringes, which is the same prediction as in the case of an aether wind. As a result, the null result from the Michelson-Morley experiment does not reject the Galilean transformation, but highlights the importance of the interaction between the environment and the photons.

There are different versions of the Michelson-Morley experiment: the Kennedy-Thorndike experiment (Kennedy and Thorndike, 1932) [13], which used different arm lengths between the mirrors and the beam splitter, and the one-way Michelson-Morley experiment (e.g. Gurzadyan, 2005) [14], which used star light as the source. By using the same approach shown in this section, the results of these experiments can all be explained by the relative speed of photons with respect to the observer and the interaction between the photons and the frame. For example, to explain the Kennedy-Thorndike experiment, we just need to replace the arm length $l$ with $l_{1}$ for the top mirror and $l_{2}$ for the right mirror to obtain the travel time for both rays:

$$
t 1^{\prime \prime}=\frac{2 l_{1}}{c} \text { and } t 2^{\prime \prime}=\frac{2 l_{2}}{c}
$$

Since $t 1^{\prime \prime} \neq t 2$ ", there will be a time difference for the photons coming back to the splitter so there will be interference fringes. However, as the above equations show, the time for both photons to come back to the beam splitter is related only to the lengths of the arms and the light speed in a vacuum, so the speed of the frame affects neither $t 1$ "nor $t 2$ ". Consequently, if the apparatus rotates, $t 1$ "and $t 2$ "will not change, thus resulting in no shifts in the fringes.

\section{The Significance of the Hoek Experiment}

In the previous section, we explained the null results of the MM and KT experiments by utilizing the almost unitary refractive index for the medium in the experiments. What if the MM and KT experiments were done with the measure- 
ment chamber filled with a medium with a high refractive index such as water? The result of such an experiment should also be negative, as suggested by the Hoek (1868) [15] experiment.

Hoek (1868) [15] used a simplified Fizeau (1851) [1] apparatus: the U-shaped tube of flowing water was replaced by a segment of glass tube filled with stationary water, so only one section of the light rays passed through the water. Ignoring the motion of the earth, the water is stationary and thus there is no dragging effect, so the interference pattern would not change if one changed the direction of the apparatus. In reality, the earth rotates on its axis and orbits the sun, so the moving earth frame will pass the speed to the water tube. If the apparatus is placed in different directions, the speed of the water tube along the direction of the light rays varies and this may affect the interference pattern. However, the Hoek experiment showed that the interference pattern did not depend on the speed of the water tube indicated by the direction of the apparatus. This result is in stark contract with the result from the Fizeau water tube experiment, which categorially showed that water speed can affect the speed of light.

To explain the result of the Hoek experiment, we need to dig a bit deeper into Equation (11): why does it show that the speed imparted to photons depends on the refractive index? We can interpret Equation (11) in two steps. First, photon matter scattering imparts the speed fully to the photons, so the rule is the same, independent of the refractive index of the medium. Then, the frequent collisions with medium particles slow down the photon speed. This slowing down can be explained by the extra distance or time taken for light to travel in the medium as a result of the collisions: the collisions with the particles of the medium make photons travel in zig-zag paths (or photons may pause during the collisions according to the photon absorption-reemission hypothesis), so the distance (or time) of photon travel is greater than the straight path that occurs in a vacuum. The denser the medium, the more collisions encountered by the photons and the longer the distance (or time) they have to travel. In this reasoning, the seemingly lower speed of light in a medium is due to this uncounted longer distance (or time of pause) of light travel. The actual speed of light travel in the medium is the same as that in a vacuum. This reasoning is consistent with the fact that once the light emerges from the medium to a vacuum, it immediately resumes its speed in a vacuum.

Following the above discussion, we can conclude that when we perceive that light speed in a medium reduces by the factor of the refractive index compared with the speed in vacuum, the unseen truth may be that photons travel at the same speed as in a vacuum and take on the full speed of the frame through photon matter scattering, but the travel distance (or time) increases by a factor of its refractive index. As such, we can modify Equation (12) to obtain the result for the Hoek experiment:

$$
t^{\prime}=\frac{n_{0} l}{c-v+v}+\frac{l}{c+v-v}=\frac{\left(n_{0}+1\right) l}{c}
$$


The above equation shows that light travel time does not depend on the speed of the earth frame, so the result of the experiment should not be affected by the speed of the frame. If we apply the same explanation to a MM or KT experiment with the main chamber filled with water, we should conclude that the interference pattern should not depend on the direction of the apparatus.

The other way of understanding the null results of the Hoek experiment is to view the experiment from different reference frames, as shown in Figure 5.

Panel (a) shows that the experiment is conducted and observed by a person who is stationary in the moving experimental frame of speed $u$. He/she will observe that the light travels at speed $c$ before entering the water tube and then travels at speed $v_{0}=c / n_{0}$. However, if the experiment is observed by a person in a rest frame, as shown in Panel (b), based on Galilean transformation, he/she will observe that the light travels at speed $c+u$ before entering the water tube and

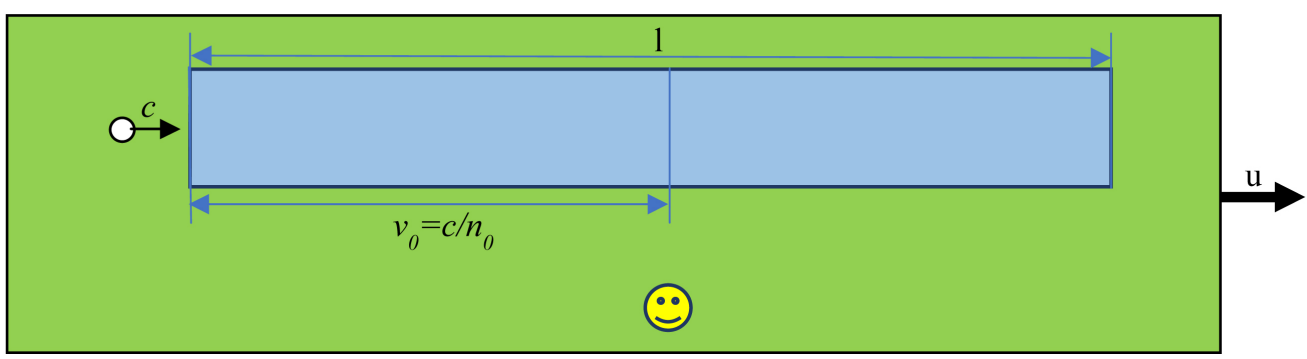

(a)

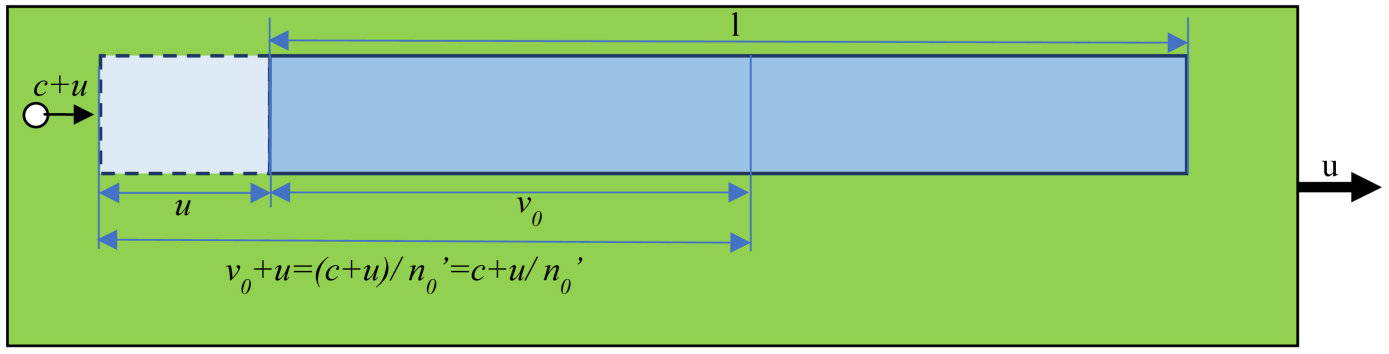

(b)
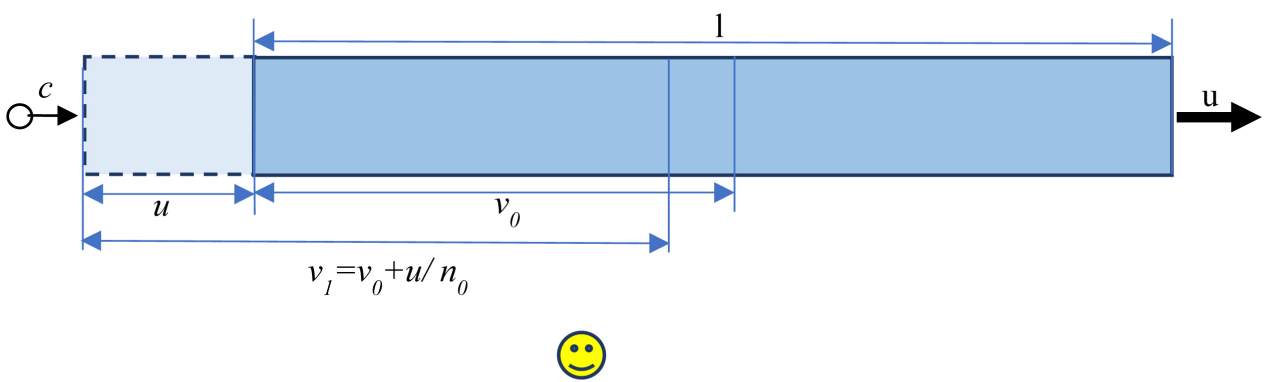

(c)

Figure 5. Hoek experiment result viewed from different reference frames. (a) Both experiment and observer in the moving frame; (b) Experiment in moving frame, observer in the rest frame; (c) Both experiment and observer in the rest frame. 
then travels at speed $v_{0}+u$. It is easy to understand why the speed of photons prior to entering the water tube increases by the amount of $u$ : the interaction (scattering) with the experimental frame imparts the frame speed to the photons. However, how can the scattering with water molecules in the tube impart fully the speed $u$ ? This seems contradictory to Equation (11), which shows that the imparted speed should be $u / n_{0}$.

The mystery is solved by examining the definition of refractive index $n_{0^{\circ}}$. For the person in panel (a), we have $n_{0}=c v_{0}$. For the person in the rest frame in panel (b), however, the refractive index becomes $n_{0}^{\prime}=(c+u) /\left(v_{0}+u\right)$. From this we can derive:

$$
v_{0}+u=(c+u) / n_{0}^{\prime}=v_{0}^{\prime}+u / n_{0}^{\prime}
$$

The above equation shows that if we use the right refractive index $n_{0}^{\prime}$, Equation (11) is still valid. This equation can be interpreted as follows: the photon can pick up the full speed of the matter through scattering but the refractive index acts as a discount factor, which discounts not only the newly picked up speed but also the original light speed.

Next, we can calculate the time for the light to travel through the water tube of length $I$. In panel (a), the light travels in water at speed $v_{o}$, so the travel time is:

$$
t_{a}=l / v_{0}
$$

In this travel time, the frame will move a distance:

$$
d=u * t_{a}=u l / v_{0}
$$

So, the time for the light to travel through the water tube in panel (b) is:

$$
t_{b}=\frac{l+d}{u+v_{0}}=\frac{l+u l / v_{0}}{u+v_{0}}=l / v_{0}
$$

We see that $t_{a}=t_{b}$ and both are independent of the frame speed, so the Hoek experimental results should be insensitive to the direction of the apparatus and should be the same viewed from different reference frames.

For comparison, we also add a scenario where the experiment is conducted and observed in the rest frame with the water tube moving at speed $u$, as shown in panel (c). This scenario is similar to the Fizeau experiment of water flow but, unlike the latter experiment, the number of photon molecule collisions do not change. Note that in panel (c), the speed of light before entering the water tube is $c$ and thus the traditional refractive index relation holds. As such, we can apply Equation (11) to obtain the speed of light in water as $v_{l}=v_{0}+u / n_{0}$. This speed is with respect to the rest frame. The relative speed with respect to the water tube is:

$$
v_{2}=v_{1}-u=v_{0}+\left(u / n_{0}\right)-u
$$

So, the time for the light to travel through the water tube is:

$$
t_{c}=\frac{l}{v_{2}}=\frac{l}{v_{0}+\left(u / n_{0}\right)-u}
$$


Since $t_{c}$ depends on the speed of water tube $u$, we expect that a change in $u$ will cause a shift of fringes if the water tube in the Hoek experiment is moving in the experimental frame. The different results for panels (b) and (c) highlight the different consequences of the movement of a frame and the movement of the water tube in a rest frame. These consequences result from the different speeds of light prior to entering the water tube.

The importance of the Hoek experiment has not been fully realized to date. It provides clear evidence that the speed of the inertial frame does not affect the experimental result, i.e. the equivalence of inertial reference frames. From another perspective, it shows that it is impossible to detect the motion of the inertial frame while being within the frame, so we are unable to detect absolute motion/rest.

\section{Evidence from Sagnac Experiment}

The 1899 Sagnac experiment (Sagnac, 1899) [16] is an interesting case because both its light source and detector move at the same speed in a closed circle.

The light propagation in the Sagnac experiment is shown in Figure 6. A light source and a phase detector are mounted on $\mathrm{A}$, which in turn is mounted on a turning disc placed on a fixed base. In a symbolic fashion, the light travels in the blue circle of circumference $s$ (which is realized through a number of reflecting mirrors). We illustrate two sets of light sources and detectors on A so that the lights travelling in opposite directions can be shown separately in different circles. Both light 1 and light 2 start at the light source and end at the detector. They travel on the same track during the experiment (although it is shown as different circles in Figure 6), so the circumferences they travel are the same.

If the disc is stationary on the base, both light beams travel the same circumference $s$ at the same speed $c$, so they will arrive at the detector at the same time: $t=s / c$. As a result, there will be no phase difference and thus no interference pattern. If the disc starts to spin at speed $v$, the experimental results can test different theories and assumptions.

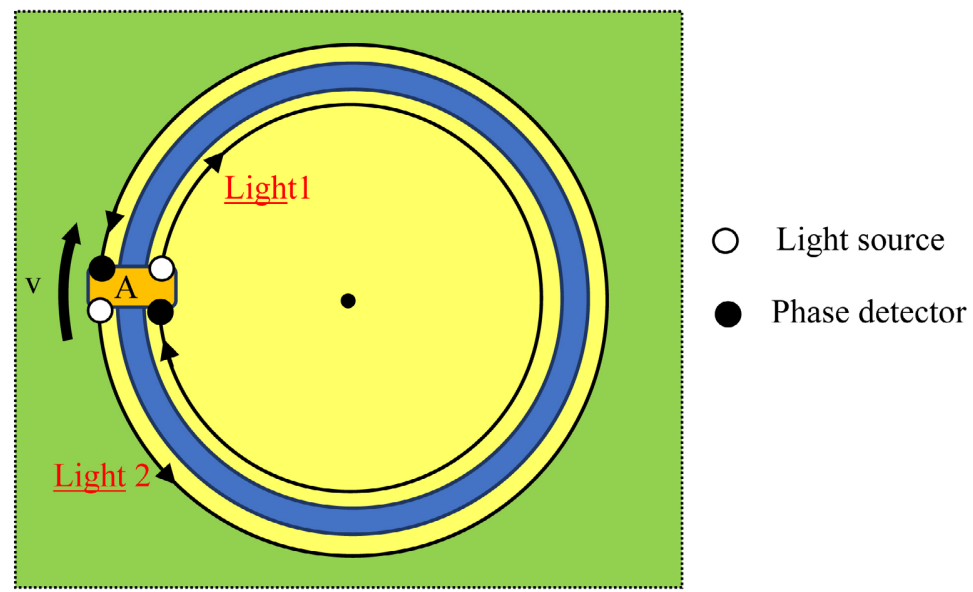

Figure 6. A schema of the Sagnac experiment. 
According to the emission theory of Ritz (1908) [5] [6], light is emitted particles that carry the speed of the light source, therefore the speeds of light 1 and light 2 with respect to the stationary base should be $c+V$ and $c-V$, respectively. The relative speed between light 1 and the detector is $c+v-V=\mathcal{c}$, so the time for light 1 to reach the detector is $t 1=s / c$. Similarly, the relative speed between light 2 and the detector is $c-v+V=c$, so light 2 arrives at the detector after time $t 2=s / c$. Apparently, $t 1=t 2$. Since both lights should arrive at the detector at the same time, no interference pattern would occur.

The relativity theory assumes that light speed in a vacuum is independent of the light source, so the speed of both lights with respect to the stationary base should be the same speed $c$. However, the theory also assumes that the speed of light is the same for any reference frame, so the speed of light with respect to the detector should be the same as that with respect to the stationary base. As such, the speed of light 1 and light 2 with respect to the detector should also be $c$, so both lights should arrive at the detector simultaneously due to the same travel time $t=s / c$. As a result, no interference fringe will occur.

If the speed of light is independent of the light source but relevant to the speed of the observer, light 1 and light 2 should have the same speed $c$ with respect to the stationary base, but the relative speed with respect to the detector should be different. The relative speed of light 1 with respect to the detector should be $c-v$, so the time for light 1 to reach the detector is $t 1=s /(c-v)$. Similarly, the relative speed of light 2 with respect to the detector should be $c+V$, and the time for light 2 to reach the detector is $t 2=s /(c+v)$. As light 1 and light 2 arrive at the detector at different times, an interference pattern will appear.

The Sagnac experiment showed that the interference pattern occurred when the disc started to spin. The only explanation consistent with the Sagnac experiment result is that the speed of light relative to the observer is independent of the speed of the light source but is dependent on the speed of the observer. However, this explanation is rejected by the null result from the Michelson-Morley experiment. This result categorically rejects both the emission theory and the relativity theory. For the relativity theory, Einstein argued that the Sagnac experiment is not done in an inertial frame and thus special relativity is not applicable. The general relativity framework can indeed explain the Sagnac experiment, so the only theory that can explain all existing experiments is the relativity theory.

Using the revelations from the Fizeau water tube experiment, we can crystalize Einstein's argument and can explain more clearly and directly the seemingly contradictory positive result from the Sagnac experiment and the negative result from the Michelson-Morley experiment. In both experiments, the photon matter scattering can fully impart the speed of the reference frame to the photons. As explained previously, this leads to the negative result of the Michelson-Morley experiment. However, the speed of the reference frame in the Sagnac experiment keeps changing, i.e. it is a non-inertial frame. Since the scattering process needs time to impart the speed to the photons, it is hard for the photons to keep up with the changing speed of the reference frame $\tilde{v}$, so only part of the changing 
speed can be imparted to the photons. Namely:

$$
\Delta \tilde{v}=\frac{f \tilde{v}}{n_{0}}, 0<f<1
$$

As such, the speed for light 1 and light 2 with respect to the detectors are, respectively,

$$
\begin{aligned}
& v_{1}=c-\Delta \tilde{v}+\tilde{v}=c+\tilde{v}-\frac{f \tilde{v}}{n_{0}} \\
& v_{2}=c+\Delta \tilde{v}-\tilde{v}=c-\tilde{v}+\frac{f \tilde{v}}{n_{0}}
\end{aligned}
$$

Due to the less-than-unitary $f$, the two speeds $V_{1}$ and $v_{2}$ are different even though the refractive index is 1 . As a result, the two photons come back to the detectors at different times and generate interference fringes. If one can measure the displacement of the fringe shift in the Sagnac experiments, one can calculate $f$, the fraction of varying speed transferred to the photons.

\section{Conclusions}

By re-examining the Fizeau water tube experiment, this paper discovers the rule for photon matter scattering, which shows that scattering imparts to photons an amount of speed that is equal to the speed of the matter times the inverse of the refractive index of the medium. The re-examination of the MM, KT and Hoek experiments further shows that photon matter scattering can impart the full speed of the matter to the light but then the new speed of light is subject to the discount of the refractive index of the medium. The analysis of the Sagnac experiment reveals that photon matter scattering can only partially impart the speed of a non-inertial frame to the photons, which supports Einstein's argument that special relativity is applicable only to the inertial frame. In short, the paper is able to consistently explain the major historical experiments on the constant speed of light and reveals the classical mechanism that leads to the constant speed of light for all inertial reference frames.

If one has not read the paper carefully, however, one may conclude that what the paper uncovered is essentially the totally dragged theories (e.g. Stokes, 1845 and Hertz, 1890) [3] [4], or the emission theory by Ritz (1908) [5] [6]. Some ideas that are similar to these theories may appear in this paper, but the mechanism revealed in this paper is totally different from previous theories. For example, this paper views light as photons emitted from a light source, so it could be called a light emission theory or a particle theory of light. However, Ritz's emission theory assumes that light carries on partially or fully the speed of the light source, which has been disproved by experiments and observations. The current paper assumes that light speed is independent of the state of the light source, so it fundamentally differs from Ritz's emission theory. The same can be said for the total dragging theories. The current paper discovers that photons can absorb the speed of the inertial frame through photon matter scattering. This does con- 
cur with the idea of the total dragging theories, which provide no mechanism for total dragging and assume that the total dragging will occur unconditionally. This assumption is proven wrong by the Fizeau experiment. The mechanism revealed in the current paper describes quantitatively how the speed of an inertial frame is imparted to the photons and why the refractive index of medium matters. It is easy to tell that the mechanism in this paper is different from the total dragging theories or Ritz's emission theory. While these two types of theories could not explain the results of the Fizeau experiment, the current paper can explain all experiments regarding the constant speed of light.

\section{Conflicts of Interest}

The author declares no conflicts of interest regarding the publication of this paper.

\section{Acknowledgements}

The author thanks Michael Evans for useful comments and suggestions.

\section{References}

[1] Fizeau., A. (1851) Sur les hypotheses relatives a l'ether lumineux, et sur une experience qui parait demontrer que le mouvement des corps change la vitesse avec laquelle la lumiere se propage dans leur interieur. Comptes Rendus de l'Académie des Sciences, 33, 349-355.

[2] Michelson, A.A. and Morley, E.W. (1887) On the Relative Motion of the Earth and the Luminiferous Ether. American Journal of Science, 34, 333-345.

https://doi.org/10.2475/ajs.s3-34.203.333

[3] Stokes, G. (1845) On the Aberration of Light. Philosophical Magazine, 27, 9-15.

[4] Hertz, H. (1890) Ueber die Grundgleichungen der Elektrodynamik fur ruhende korper. Annalen der physic und Chemie, 277, 369-399. https://doi.org/10.1002/andp.18902771102

[5] Ritz, W. (1908) Recherches critiques sur l'electrodynamique general. Annales de Chimie et de Physique, 8, 145-275.

[6] Ritz, W. (1908) Recherches critiques sur les theories electrodynamique de C. Maxwell et de H-A Lorentz. Archives des sciences physiques et naturelles, 26, 209-236.

[7] Fresnel, A. (1818) Lettre d'Augustin Fresnel a Francios Arago sur l'influence du movement terrestre dans quelques phenomenes d'optique. Annales de Chimie et de Physique, 9, 57-76.

[8] Michelson, A. and Morley, E. (1886) Influence of Motion of the Medium on the Velocity of Light. American Journal of Science, 31, 377-386. https://doi.org/10.2475/ajs.s3-31.185.377

[9] Thomson, J. (1880) On the Maxwell's Theory of Light. Philosophical Magazine, 9, 284-291. https://doi.org/10.1080/14786448008626840

[10] Lorentz, H. (1892) La theorie electromagnetique de Maxwell et son application aux corps mouvants. Archive Neerl, 25, Article No. 363.

[11] Lorentz, H. (1895) Versuch einer theorie der elektrischen und optischen Erscheinungen in bewegten Korpern. LBrill, Leiden. 
[12] Laue, M. (1907) Die Mitführung des Lichtes durch bewegte Körper nach dem Relativitätsprinzip. Annalen der Physik, 328, 989-990.

[13] Kennedy, R.J. and Thorndike, E.M. (1932) Experimental Establishment of the Relativity of Time. Physical Review, 42, 400-408. https://doi.org/10.1103/PhysRev.42.400

[14] Gurzadyan, V.G., et al. (2005) Probing the Light Speed Anisotropy with Respect to the Cosmic Microwave Background Radiation Dipole. Modern Physics Letters A, 20, 19-28. https://doi.org/10.1142/S0217732305016294

[15] Hoek, M. (1868) Determination de la vitesse avec laquelle est entrainée une onde lumineuse traversant un milieu en mouvement. Verslagen en Mededeelingen, 2, 189-194.

[16] Sagnac, G. (1899) Théorie nouvelle des phénomènes optiques d'entraînement de I'éther par la matière. Comptes rendus hebdomadaires des séamces de I'Académie des sciences, 129, 818-821. 